\title{
Linx
}

Revue des linguistes de l'université Paris X Nanterre

$55 \mid 2006$

Thème et thématisation

\section{Les marques formelles de la thématisation dans les langues flexionnelles, le cas de l'allemand}

Formal topicalization markers in inflected languages : the case of German.

Jean-Jacques Briu

\section{OpenEdition}

Journals

Édition électronique

URL : http://journals.openedition.org/linx/382

DOI : $10.4000 /$ linx.382

ISSN : 2118-9692

Éditeur

Presses universitaires de Paris Nanterre

Édition imprimée

Date de publication : 1 décembre 2006

Pagination : 49-60

ISSN : 0246-8743

\section{Référence électronique}

Jean-Jacques Briu, «Les marques formelles de la thématisation dans les langues flexionnelles, le cas de l'allemand », Linx [En ligne], 55 | 2006, mis en ligne le 22 février 2011, consulté le 01 mai 2019. URL : http://journals.openedition.org/linx/382 ; DOI : 10.4000/linx.382 


\title{
Les marques formelles de la thématisation dans les langues flexionnelles, le cas de l'allemand
}

\author{
Jean-Jacques Briu, UMR 7114 MoDyCo, Paris $X$ \\ jjbriu@club-internet.fr
}

L'allemand est une langue à cas et un des corollaire de ce fait est une certaine souplesse dans l'ordre des mots. A contrario, la place du verbe et celle du négateur de phrase (nicht, k-, kein) sont fortement contraintes en allemand. Ces deux contraintes présentent une particularité inattendue dans l'analyse de la phrase en thème et rhème. Elles fourniraient, en effet, deux marques formelles bornant le rhème et, par conséquent, une délimitation différentielle automatique du thème. En cela, l'allemand présente une différence sensible avec beaucoup d'autres langues indoeuropéennes.

Je replacerai d'abord la question du thème et du rhème dans la dimension historique, étant donné que leur acception, dans leur perspective d'analyse et avec leur(s) critère(s) particulier(s), a fluctué depuis l'Antiquité. Nous mettrons ensuite en relief la position particulièrement intéressante qu'a prise Jean-Marie Zemb dans l'analyse de l'allemand en s'attachant à l'opposition logique Thème vs Rhème et à l'articulation du «Phème ». Ce cadre théorique ainsi défini, j'examinerai plus particulièrement la question de savoir dans quelle mesure on peut parler de thématisation. Enfin, en nous appuyant sur l'analyse de phrases allemandes, nous verrons quelles marques formelles présente le Thème de manière constante. 
Jean-Jacques Briu

\section{La dimension historique}

Pour Platon et Aristote, toute proposition relève d'une analyse logicophilosophique en deux parties: sujet et prédicat, ensemble nominal et ensemble verbal, où le « sujet » est entendu comme ce qui supporte des attributs.

Dans les grammaires générales, on maintient la division sous la forme de sujet et d'attribut: Ainsi dans Arnauld et Lancelot (1660) « [...] toute proposition enferme nécessairement deux termes; l'un appelé sujet, qui est ce dont on affirme, comme terre; et l'autre appelé attribut, qui est ce qu'on affirme, comme ronde; et de plus la liaison entre ces deux termes, est. $»^{1}$

Sujet logique et sujet grammatical (nominatif) sont souvent assimilés l'un à l'autre, même si on donne à « sujet» son sens large et premier de matière du discours, ce dont on se propose de dire quelque chose.

Hermann Paul (1880) ${ }^{2}$ reprend l'analyse dichotomique classique de la phrase en «sujet» et "prédicat»: «Toute phrase se compose d'au moins deux éléments. Ces éléments ne sont pas dans le même rapport, mais se différencient par leur fonction $»^{3}$. Ce que souligne Paul, c'est qu'ils peuvent être grammaticaux ou psychologiques, qu'ils ne coïncident pas toujours, mais que le rapport grammatical repose sur un fondement psychologique. La relation sujet - prédicat est « une association de deux représentations » ${ }^{4}$ où le prédicat suit chronologiquement le sujet et le détermine. Leur relation se présente parfois sous la forme d'un simple rapport de juxtaposition où l'on ne peut reconnaitre de sujet grammatical, en l'absence de verbe, mais seulement un sujet psychologique ; C'est une forme proverbiale commune ; par ex. : all. " ein Mann, ein Wort; viele Köpfe, viele Sinne; fr. bonne terre, mauvais chemin; point d'argent, point de Suisse; angl. like master, like man; small gains, small pains ${ }^{5}$. La distinction du sujet et du prédicat devient une opposition d'ordre psychologique. Le « sujet psychologique » d'un énoncé, à partir de la fin du $19^{\mathrm{e}}$ siècle est ce dont parle le locuteur; il préfigure le Thème (reprise du terme grec ancien), en angl. «topic ». Le « prédicat psychologique », qu'on appelle Rhème ou propos, angl. «comment» est l'information que le locuteur rapporte à ce sujet / thème.

\footnotetext{
1 Arnauld et Lancelot (1660) Grammaire générale et raisonnée, chap. I.

2 Prinzipien der Sprachgeschicbte, Halle. 10e éd., Tübingen, Niemeyer, 1995, 428 p.

3 Trad. J-J B. : «Jeder Satz besteht demnach aus mindestens zwei Elementen. Diese Elemente verhalten sich einander nicht gleich, sondern sind ihrer Funktion nach differenziert. Man bezeichnet sie als Subjekt und Prädikat.», op. cit. 124.

4 «Wir müssen daran festhalten, dass es nur darauf ankommt, dass eine Vorstellung im Bewusstsein an die andere angeknüpft ist» [fr. « Nous devons nous en tenir au simple phénomène d'association dans la conscience de deux représentations. » Trad. J-J B], op. cit. 125.

5 Trad. J-J B. : all. «Un homme, une parole; autant de têtes, autant d'avis (...) tel maittre, tel homme; petits gains, petites peines ». Paul donne une longue liste, dans trois langues, de ces formes averbales où l'énoncé se réduit à deux éléments constitutifs juxtaposés présentant un rapport de détermination. Op. cit. 125.
} 
Cette distinction apparaît aussi chez les linguistes du Cercle de Prague, par V. Mathesius dès $1929^{6}$, qui développent alors une «Perspective fonctionnelle de la phrase ». Charles Bally $(1932)^{7}$ est souvent considéré comme le premier à avoir proposé l'opposition thème vs propos:

la pensée qu'on veut faire connaitre est - nous le répétons - le but, la fin de l'énoncé, ce qu'on propose, en un mot: le propos; on l'énonce à l'occasion d'une autre chose qui en forme la base, le substrat, le motif : c'est le thème (p. 52).

L'opposition thème/rhème est d'ordre pragmatique, informationnel : elle est liée au discours; " elle permet de séparer dans un énoncé ce que l'on sait déjà (le thème) de ce qu'on apporte de nouveau (le rhème) $»^{8}$.

Dans toute analyse dichotomique de la phrase, en « sujet vs prédicat» ou en « thème vs rhème », il suffit de trouver l'un des constituants, pour être certain de trouver aussi l'autre. Et réciproquement. Pourtant, rien n'est aussi simple car trouver les limites de chacun des deux termes revient d'abord à définir leur spécificité propre, quels que soient les critères mis en œuvre. On fait alors de la phrase un système relatif de trois termes où la Phrase est l'unité supérieure comprenant deux sous-parties constituantes, le thème et rhème ; l'analyse logique formelle par soustraction semble simple :

Phrase - Rhème $=$ Thème $;$ et Phrase - Thème $=$ Rhème .

On retrouverait là une parfaite analogie avec l'analyse classique de la phrase en sujet et prédicat :

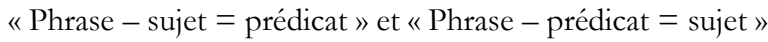

Or en plus de la spécificité des deux constituants, il est nécessaire de définir le type de rapport que l'on reconnaît entre ces constituants, entre le thème et le rhème. On peut au moins avancer avec certitude qu'il est - le plus souvent - formellement corrélatif (aucun n'existe sans l'autre) et ordonné (le thème est d'abord exprimé, le rhème se réfère ensuite à lui).

Étant donné la diversité des dénominations existant désormais : sujet vs prédicat (Paul 1880), thème vs rhème (Mathesius 1929) et thème vs propos (Bally 1932), donné vs nouveau (Halliday 1967), topic vs comment (Hockett 1958), thème vs rhème (Zemb 1968), thème vs focus (Sgall 1973), thème vs phème vs rhème (Zemb 1978), préfixe vs noyau (BlancheBenveniste 1997), il faudra, pour échapper à la confusion, identifier le plan sur lequel s'établit ce rapport: syntaxique, sémantique, logique, psychologique, pragmatique (aucun de ces plans n'est d'ailleurs forcément exclusif des autres). De même, il conviendra d'évaluer les critères et la méthode opératoire employés dans chaque analyse (information nouvelle, interrogation, dislocation, focalisation...).

\footnotetext{
${ }^{6}$ Voir Mélanges Bally, Genève, 1939 et Travaux linguistiques de Prague, I, Prague, 1964.

${ }^{7}$ Linguistique générale et linguistique française, Paris, Leroux. 4e éd., Berne, Francke, 1965.

${ }^{8}$ Chiss, J.-L. \& Filliolet, J. \& Maingueneau, D. (2001) Introduction à la linguistique française, Paris, Hachette, tome 2, p. 67-68.
} 
Jean-Jacques Briu

\section{L’opposition logique et syntaxique Thème vs Rhème... et le Phème}

Quelles sont en allemand les «marques formelles » et autres critères du langage observables et constants qui permettent de caractériser les deux notions de Thème et de Rhème?

Le germaniste français Jean-Marie Zemb ${ }^{9}$ a montré qu'il existe, de fait, dans la phrase allemande une marque (le négateur de phrase) qui borne le début du Rhème ${ }^{10}$. O. Ducrot \& J-M Schaeffer (1995) signalent que, dans Les structures logiques de la proposition allemande, (1968), " J.M. Zemb utilise l'opposition thème-rhème pour étudier la négation $»^{11}$, or c'est exactement l'inverse : c'est à partir de la négation globale, et précisément à partir de la place du négateur qu'il étudie la partition de la proposition en thème-rhème.

Un autre élément important de ses analyses est de montrer que l'ensemble du Rhème présente en allemand une structure différente de celle du thème, dans la mesure où elle est soumise à une stricte hiérarchisation du déterminant et du déterminé. Elle constitue ce qu’on appelle un «ordre récursif». Enfin, dans sa Grammaire comparative (1978), il s'attache à montrer qu'une analyse plus fine de la phrase nécessite de prendre en compte un troisième élément, le Phème, qui, à l'articulation du Thème et du Rhème, module leur relation qui est, pour lui, d'ordre syntaxique. On aurait ainsi trois séries de «marques formelles».

Dans son analyse de la «proposition allemande » publiée en $1978^{12}$, Jean-Marie Zemb abandonne, à la suite de Tesnière, les notions traditionnelles de la phrase comme la relation d'un sujet grammatical et d'un prédicat. Il appelle «proposition » la séquence qui est proposée à l'assentiment, à quoi l'on peut réagir par oui, non, peutêtre, etc. La «proposition, c'est ce qui est posé, asserté, affirmativement ou négativement (fût-ce virtuellement, dans l'interrogation) $»^{13}$. Zemb observe que l'ordre syntagmatique des termes de la proposition est dérivé d'un « ordre de base » propre à l'allemand. De quoi s'agit-il ? L'ordre de base est une structure de référence qui est abstraite, indépendante du discours et non assertée ${ }^{14}$; elle comprend trois types de données réparties entre le thème, en tête de séquence, le rhème en fin de séquence et le phème entre les deux. Prenons pour exemple la proposition suivante :

\footnotetext{
${ }^{9}$ Professeur honoraire au Collège de France, chaire de Pensée et grammaire allemandes.

10 Dans notamment: Zemb Jean-Marie (1968) Les structures logiques de la proposition allemande, Paris, OCDL, $349 \mathrm{p}$.

11 Nouveau dictionnaire encyclopédique des sciences du langage, Paris, Le Seuil, 1995, p. 543.

12 Zemb Jean-Marie (1978) Vergleichende Grammatik I, Mannheim, Dudenverlag, 897 p., voir notamment p. 387-527).

13 Vergleichende Grammatik I, p. 395. ex. : Ich kenne kein Meer, dessen Wasser nicht verseucht sind Jje ne connais pas de mer dont les eaux ne sont pas polluées]; Wenn ich mich nicht geirrt hätte, würde ich mich freuen. [Si je ne m'étais pas trompé, je serais heureux]; Diese Beispiele sollen vorläufig ausreichen. [Ces exemples doivent pour l'instant suffire]. Trad. J-J B.

14 Il l'appelle un « architaxème » (Vergleichende Grammatik I, p. 397).
} 
(1) Heute dürtte das Wetter in derNormandie leider unbeständig sein.

(1a) Aujourd'hui devrait la météo sur la Normandie hélas incertaine être

(1b) Aujourd'hui la météo sur la Normandie devrait hélas être incertaine.

Cette proposition aurait la structure logique (simplifiée) suivante :

\author{
das Wetter \\ heute \\ in der Normandie \\ -ü -te présent
}

\author{
leider \\ affirmation \\ subjonctif
}

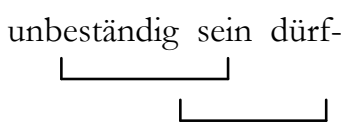

RHEME
PHEME

THEME

1.1. Examinons le Rhème. Le noyau du Rhème est toujours un verbe. Il est précédé des compléments rhématiques (all. Ergänzungen) qui forment une suite de déterminations, le déterminé ultime étant le noyau verbal. Exemple :

(1c) / unbeständig sein dürfen / = unbeständig [sein (dürfen)]; fr. incertain [être (devoir)]

Les éléments du rhème sont dans une relation hypotaxique, strictement hiérarchisés à partir du verbe qui est toujours le noyau de la suite rhématique. Exemple :

(1d) / schnell in die Stadt fahren / = schnell [in die Stadt (fahren)]

fr. rapidement [ en ville (se rendre)]

On observe sur les plans syntagmatique et syntaxique - c'est une donnée didactique de première importance ! - que l'ordre français est exactement l'inverse de celui de l'allemand. Sur le plan logico-sémantique, le Rhème signifie essentiellement une «idée », une « notion », une signification « pure 115 .

1.2 Le Thème. En face du Rhème, on trouve, dans l'ordre de base, le Thème, c'est-à-dire « l'ensemble des arguments » (p. 401). Soit pour l'exemple (1) :

(1e) beute + das Wetter... + in der Normandie $+\ddot{u}$-te (temps présent)

(1f) fr. aujourd'hui + la météo...t + en Normandie + -rai (temps présent)

Alors que les éléments du Rhème sont strictement biérarchisés (hypotaxe), les éléments du Thème, eux, sont seulement co-ordonnés (parataxe). Ensemble, ils délimitent,

\footnotetext{
15 Zemb reconnait cependant que dans les rhèmes, on ne trouve pas que des abstraits, p. ex. «aus dem Norden stammen, nach Hamburg fliegen, nach Canossa reisen » (fr. être originaire du nord ; prendre l'avion pour Hambourg; aller à Canossa), p. 399 ; à moins de considérer que l'abstraction, c'est l'idée de provenance ou de destination.
} 
Jean-Jacques Briu

ils désignent la représentation d'une "portion de réalité » à laquelle on attribue, d'une manière ou d'une autre (déterminée par le Phème), un prédicat ou Rhème.

1.3. Le Phème. Affinant son analyse, Zemb observe que «Dans l'ordre des termes de la proposition allemande, un espace sépare le thème du rhème » (403). Il s'agit d'un troisième élément constitutif, qui existe de la même façon en français par exemple. Le phème n'est pas symétrique au thème et au rhème : il ne se situe pas sur le même plan et n'a pas la même fonction. Le phème porte sur la relation entre le thème et le rhème, il la module. On y trouve quatre classes d'éléments dans un ordre hiérarchisé :

a) le négateur «de phrase »: «nicht, nicht mehr, nie...» (fr. ne pas, ne plus, jamais...) ; l'affirmation (plus fréquente) n'est marquée que par l'absence de négation/ de négateur ;

b) le mode : indicatif ou subjonctif, mais également les modalisateurs indiquant un degré de probabilité : «vielleicht, gewiß, bestimmt, sicher... » (fr. peut-être, sûrement, certainement, assurément...);

c) des appréciatifs : "notwendigerweise, möglicherweise, leider, natürlich, offensichtlich...» (fr. nécessairement, éventuellement, malheureusement, naturellement, manifestement, visiblement...);

d) des particules modales à valeur illocutoire : «aber, auch, bloß, schon, ja, mal...» (en fr. l'emploi de ces particules est restreint, leur traduction très variable en contexte et situation).

Résumons l'essentiel : la proposition relève d'un « acte prédicatif » par lequel on réunit Thème et Rhème.(403). Le sujet parlant opère un jugement qui réunit d'une part un Rhème, une idée, et d'autre part un Thème, une représentation de réalité. Cette opération, selon l'expression de Zemb, «donne un sens aux choses et un corps aux idées ».

«L'ordre de base n'est pas une suite linéaire de termes, il ne définit pas une succession déterminée des membres de phrase»(405). Il est la combinaison d'une suite de termes qui sont rhématiques en fin de proposition, phématiques à l'articulation entre thème et rhème, et d'une liste de termes thématiques. La structure canonique de la proposition allemande correspondrait au schéma suivant, où Th = élément du thème, $\mathrm{Ph}=$ élément du phème et $\mathrm{Rh}=$ élément du rhème :

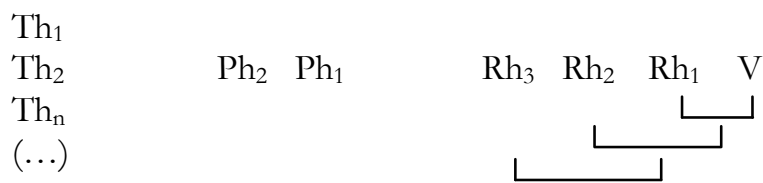

\section{Thématisation vs Thème :}

On peut avoir des phrases avec des Thèmes très riches tels que :

(2) (daß) er ihn dort damals ohne Grund (nicht aufgesucht hätte) (401).

(2a) (qu') il le là-bas à l'époque sans raison (n'avait pas cherché) 
(2b) (qu') il (ne) l'(avait pas cherché) là-bas à l'époque sans raison

Dans les discours suivis, les Thèmes sont souvent sémantiquement «assez pauvres parce que l'allocutaire est censé, dans la progression du discours, savoir à peu près de quoi il retourne »; il possède les données et les repères nécessaires ou utiles pour comprendre le locuteur. La question est de savoir, dans telle phrase d'un discours, à quelle réalité s'applique, selon certaines modalités, l'idée rhématique. Plus précisément, on se demandera si le Rhème (tout entier) convient à l'ensemble du Thème - ou bien s'il convient à un seul des éléments du Thème.

C'est dans ce dernier cas que nous comprenons d'abord le sens de «thématisation ». Si ce métaterme est bien formé, il désigne une opération, une (mise en) relation. Le locuteur sélectionnerait un élément parmi d'autres de l'énoncé - à l'exclusion, naturellement, de tout élément phématique ou rhématique.

Le plus souvent, font partie du Thème les cinq classes d'éléments suivantes : le syntagme en fonction de sujet grammatical, les pronoms (i.e. ceux de la $3^{\mathrm{e}}$ personne); les syntagmes en fonction de circonstants; des Groupes Nominaux définis; les diverses marques du temps.

Précisons qu'au syntagme fléchi au nominatif ou au nom propre invariable en fonction de sujet grammatical il convient d'associer la marque personnelle du verbe (redondante) ; on se souvient ici de l'exemple de Tesnière : filius patrem amat où il met en relation directe filius et $-t$. Toutefois, il est possible que ce syntagme en fonction de sujet grammatical soit rhématique ${ }^{16}$.

Le temps est exprimé non seulement par des morphèmes du verbe, mais aussi par des déictiques et des circonstants temporels.

Reprenons la phrase (1):

(1) Heute dürfte das Wetter in der Normandie leider unbeständig sein

(1a) Aujourd'hui devrait le temps sur la Normandie hélas incertain être

(1b) Aujourd'hui, le temps sur la Normandie devrait hélas être incertain.

Quel est le terme « thématisé » ? Quel est l'objet de la thématisation ? La bonne question revient à se demander à quel élément convient l'idée "unbeständig sein », « être incertain ». Au préalable, il a fallu identifier cette idée, qui constitue le contenu du Rhème. L'opération est facilitée par des repères formels : son seuil est ici explicitement marqué en allemand par l'appréciatif leider (fr. bélas), mais implicitement par l'absence de négateur. De même, dans la phrase française, la place de "hélas» est aussi significative comme repère rhématique puisque son déplacement donne des énoncés de signification différente :

(3) Hélas, aujourd'hui, le temps sur la Normandie devrait être incertain.

16 ex. (daß) morgen vielleicht Besuch kommt (demain peut-être de la visite viendra; Demain, il se peut qu'ily ait de la visite) trad. J-J B ;

(daß) vor einer solchen Theorie notwendigerweise jeder zurückschreckt (Devant une telle théorie, nécessairement, chacun recule, effrayé) trad. J-J B. 
Jean-Jacques Briu

(3') Aujourd'hui hélas, le temps sur la Normandie devrait être incertain.

(3”) Aujourd'hui le temps, sur la Normandie hélas, devrait être incertain.

Les candidats thématiques sont heute et das Wetter ou das Wetter in der Normandie (fr. aujourd'hui, le temps, le temps sur la Normandie). Un premier critère opératoire ordinairement utilisé est de formuler à partir du Rhème une question ouverte et d'élire l'élément qui fournit une réponse acceptable : qu'est-ce qui devrait «être incertain »? Quand / Où devrait-il planer une incertitude? Trois questions, trois réponses possibles. A-t-on alors autant de Thèmes ? Un second critère est le test de dislocation à l'aide de « c'est... que »; or l'opération est possible avec nos trois candidats :

(4) c'est aujourd'hui que le temps sur la Normandie devrait être incertain.

(4') c'est le temps qui devrait être incertain aujourd'hui sur la Normandie.

(4") c'est le temps sur la Normandie qui devrait être incertain aujourd'hui.

Les deux critères de la question ouverte et de la dislocation souvent utilisés pour le repérage du thème en français semblent peu concluants. On emploie encore fréquemment un troisième critère dit de détachement à gauche, qui permet de distinguer le thème du sujet syntaxique; le thème détaché étant ensuite repris par un pronom clitique sujet. C'est ce dernier qui fournit une marque formelle de limite droite de l'ensemble thématique. Cela donnerait pour (1b) :

(1b) Aujourd'hui, le temps sur la Normandie devrait hélas être incertain.

(1c) Aujourd'hui, le temps sur la Normandie, il devrait hélas être incertain.

Toutefois, «Aujourd'hui, le temps sur la Normandie» est une donnée exacte mais incomplète : il faut lui ajouter «-t» (marque de la personne sujet) et le «temps présent» (associé à «aujourd'hui »). En conclusion, le clitique est une limite formelle approximative.

$\mathrm{Au}$ lieu d'utiliser les trois critères que nous venons de décrire, un (bon) germaniste procèdera autrement : il considèrera que l'idée rhématique de variabilité (" unbeständig sein») convient - sur le mode positif (pas de négateur), avec une faible probabilité (《dürfte») et à regret (《leider») - à une réalité définie par un ensemble de plusieurs repères concomitants et solidaires, à savoir : une part de réalité, une donnée de lieu et une donnée de temps (« heute» + présent). Sa méthode consiste à partir du rhème formellement marqué, puis d'extraire le phème lui aussi formellement marqué pour trouver comme reliquat l'ensemble des éléments du thème.

$\mathrm{Si}$ tous les éléments du thème sont toujours nécessairement donnés comme le Thème, alors la thématisation comme l'élection par le locuteur d'un élément thématique particulier ne se pose pas, et la thématisation est sans objet. Dans ce cas, nous serions déjà au bord de conclure. Mais nous le ferions trop hâtivement sans doute, car il reste à examiner plus avant au moins la question des «marques formelles » du Thème ou des éléments thématiques. 


\section{Quelles marques formelles présente le Thème?}

Nous avons précédemment indiqué que dans l'ordre de base, les termes rhématiques constituent en allemand une suite en fin de proposition, tandis que les termes thématiques forment une liste en tête de séquence et qu'ils précèdent les éléments phématiques.

Dans l'ordre de distribution syntagmatique, la fin du Thème est bornée par le premier terme phématique. On aura ainsi la succession type maximale suivante :

$\{$ Th, Th ...\} [Particule modale + Appréciatif + Modalisateur + Négateur] $\left(\ldots R h_{2} \mathbf{R h}_{1} \mathbf{R h}_{0}\right)$

Sur le plan fonctionnel, à la fin du Thème, qui a une fonction désignative, apparait ainsi un marquage par un terme illocutoire et/ou un jugement de valeur et la négation / affirmation. Ensuite vient le Rhème. Prenons un nouvel exemple :

(5) (weil) der Kerl / ja zum Glück wohl nicht / so dumm ist, wie er aussiebt.

(5') (car) le type, / bien sûr, et heureusement, manifestement ne pas / aussi bête est qu'il en a l'air.

(6") (car) le type, / bien sûr, et heureusement, n'est manifestement pas / aussi bête qu'il en a l'air.

Le thème comprend les éléments : der Kerl... -t; le temps présent. (le type... -t; présent).

Le rhème est constitué de : so dumm, wie er aussieht, sein (être aussi bête qu'il en a l'air).

Le phème contient ici trois éléments : ja / zum Glück / wobl nicht (bien sûr, heureusement, manifestement pas).

La première place syntaxique de la proposition (409), c'est-à-dire la première place avant le verbe dans la phrase déclarative, n'est pas forcément celle d'un élément thématique. En allemand, cette place ne peut être occupée que par un seul groupe syntaxique. Il est thématique le plus souvent, accentué ou non. Mais d'autres éléments situés dans le verbe (le temps) et après le verbe peuvent également être des éléments thématiques. Dans les exemples suivants, l'ensemble occupant la première place en allemand est souligné. J'ai essayé dans la traduction française de conserver le même ensemble à la première place ; on voit qu'il existe plusieurs possibilités équivalentes en français avec un ordre des mots différents face à une seule possibilité correcte et claire en allemand :

(6) Das habe ich nicht gewollt.

(Ça, je l'ai pas voulu. / Je n'ai pas voulu cela. / Ce n'est pas ce que j'ai voulu.)

(7) Heute muß das Kapitel werden.

(Aujourd'hui, ce chapitre doit être fini. / Il faut finir ce chapitre aujourd'hui.) 
Jean-Jacques Briu

(8) Dieses Problem ist gar nicht so leicht.

(Ce problème n'est vraiment pas si simple.)

(9) Aus Versehen hat er ihn nicht gegrüßt.

(Par distraction, il ne l'a pas salué. / Distrait, il ne l'a pas salué.)

En tête de phrase, on peut avoir un terme rhématique, accentué ou non ; il est souligné. J'ai essayé dans la traduction française de conserver l'ensemble correspondant à la première place :

(10) An die Alster kehrt er gern zurück.

(Sur les bords de l'Alster, il retourne volontiers. / Il aime retourner les bords de l'Alster.)

(11) Einfach umfallen wird er kaum.

(«Simplement chavirer, il le fera guère.»/ Chavirer comme ça, il y a peu de chances que ça lui arrive.)

(12) Auf stieg der Strabl.

(Tout droit, il est monté le rayon. / Il est monté tout droit, le rayon.)

(13) Dumm ist das nicht.

(« Idiot est cela pas ». Pour être idiot, ce n’est pas idiot. / Non, c’est pas idiot!)

Un terme phémique (appelé encore par certains «adverbe de phrase ») peut aussi occuper la première place, qu'il soit accentué ou non - à la seule exception de la particule modale qui, en outre, ne peut pas être accentuée :

(14) Vielleicht wird es schön.

(Peut-être (qu') il fera beau / Il se peut qu'il fasse beau.)

(15) Zweifellos stammt der Sohn von seinem Vater ab.

(Sans aucun doute, le fils vient de son père. / Sans aucun doute, c'est le fils de son père.)

(16) Natürlich werden Lügner nicht die Wabrheit sagen.

(« Naturellement, diront des menteurs pas la vérité.» / Naturellement, des menteurs ne diront pas la vérité.)

(17) Nicht habe ich behauptet, dieser Kerl sei ein Idiot.

(« Ne pas j’ai affirmé ce type était un imbécile.» / En aucune façon je n'ai affirmé que ce type était un imbécile.)

(18) Noch ist Polen nicht verloren.

(«Encore est la Pologne pas perdue. » / Pas encore, la Pologne n’est pas encore perdue.) 
La catégorie du "défini» (représentée notamment par des actualisateurs, des déictiques) est une caractéristique assez constante des termes thématiques, en opposition à « l'indéfini » du terme rhèmatique - ou du «défini » lexicalisé du Rhème ; de même en français : prendre la mouche, donner le bonjour, dire la vérité, etc. Ici, l'analyse requiert souvent une prise en compte du contexte ou/et des pré-requis ou/et de la situation.

L'accentuation n'est pas forcément une marque formelle d'identification thématique. A moins que le Thème ne se réduise à un seul élément accentué, mais il faudrait encore lui ajouter le repère temporel toujours donné sur le verbe au moins. Sinon, l'accentuation a pour fonction de focaliser un élément thématique particulier parmi d'autres, mais laisse entière la solidarité syntagmatique des autres éléments thématiques. Quoi qu'il en soit, on retiendra que l'accentuation n'établit aucune hiérarchie ni aucune priorité parmi les divers éléments du Thème.

$\mathrm{Si}$ le Thème n'est pas forcément majoré par l'accentuation, il n'est pas davantage minoré dans la phrase négative. En fait, le thème n'est pas nié. Même dans le cas où la phrase contient une négation globale, même si «le prédicat est négatif ", comme on dit, l'ensemble thématique n'en reste pas moins une donnée, une réalité positive. En d'autres termes, le négateur global affecte exactement la relation ThèmeRhème (et pas la phrase en soi), mais il n'efface ni ne réduit en rien la valeur des réalités que désigne le Thème.

Les critiques que l'on peut adresser à la marque formelle que fournit le phème en allemand concernent avant tout la difficulté de délimitation et donc d'identification du rhème et du thème qui existerait en l'absence de tout élément phémique. L'absence de négateur global. notamment serait un important inconvénient, d'autant que les phrases positives sont de loin plus fréquentes dans le langage que les phrases négatives. Il reste toujours possible de nier globalement la phrase (i.e. le rapport syntaxique entre le rhème et le thème), mais cette opération révèle parfois plusieurs possibilités liées à plusieurs places possibles du négateur. Laquelle retenir ? Celle qu'autorise le mieux les liens sémantiques et logiques avec le contexte et la situation où apparaît la phrase.

La question se pose sans doute de savoir si les observations faites sur l'allemand et la méthodologie que l'on a déduite est transposable par exemple au français. On fera valoir que l'ordre des mots n'est pas très souvent le même, en particulier la place du négateur, même si l'on néglige «ne » et si l'on prend seulement en compte "pas ». On a pu le remarquer sur les traductions littérales et autres que j'ai données pour chaque exemple allemand. Ma réponse sera que si l'on admet que les exemples originaux et traduits sont parfaitement équivalents quant à la signification, il est bien difficile de soutenir que les éléments désignatifs, définis du thème seraient différents dans une langue et dans l'autre, que les éléments rhématiques n'apportent pas la même idée relativement au même thème.

Même si les observations contrastives sur le français et l'allemand permettent d'observer des différences d'ordre et de distribution des marques formelles entre les éléments thématiques, phématiques et rhématiques, l'identification de ces éléments ne reste-t-elle pas la même dans les deux langues? Reprenons l'exemple (1) : 
Jean-Jacques Briu

(1) Heute dürte das Wetter inderNormandie leider unbeständig sein.

(1a) Aujourd'hui devrait la météo sur la Normandie hélas incertaine être

(1b) Aujourd'hui la météo sur la Normandie devrait hélas être incertaine.

L'ensemble des données désignatives constituant le Thème sont bien les mêmes : dans (1) : \{das Wetter... Ø; heute ; in der Normandie ; présent $\}$

dans (2) : \{la météo...-t ; aujourd'hui ; sur la Normandie ; présent

Les données abstraites constituant le Rhème sont aussi les mêmes :

dans (1) : (unbeständig sein dürf-)

dans (2) : (devoir être incertaine)

Les éléments du Phéme sont eux aussi similaires :

(1) : (leider subjonctif affirmation)

(2) : (hélas condititonnel affirmation).

D'aucuns pourront bien considérer que le modèle zembien se réduit au plan de la syntaxe ou qu'il ne trouve sa validité que dans une logique sémantique. Personne ne peut nier pourtant que ce modèle théorique rend compte de deux fonctions essentielles de la proposition assertée : la fonction désignative des percepts d'un côté et la fonction conceptuelle des idées de l'autre. Pour être une relation syntaxique, la relation entre Thème et Rhème n'en est pas moins prédicative. Le Phème qui spécifie cette relation exprime divers rapports entre le locuteur et son énoncé de même qu'entre le locuteur et l'allocutaire et rend compte ainsi de la dimension énonciative / pragmatique de l'énoncé.

Les marques formelles propres au Rhème et au Thème, celles que fournissent comme seuils délimitatifs les éléments du Phème, dont le négateur global nicht, / k- / kein-sont des faits de langue constants, observables et incontestables. 